\title{
Coding of Communication Calls in the Subcortical and Cortical Structures of the Auditory System
}

\author{
D. ŠUTA ${ }^{1,2}$, J. POPELÁŘ ${ }^{1}$, J. SYKA ${ }^{1}$ \\ ${ }^{1}$ Department of Auditory Neuroscience, Institute of Experimental Medicine, Academy of Sciences \\ of the Czech Republic, Prague, ${ }^{2}$ Department of Medical Biophysics and Medical Informatics, Third \\ Faculty of Medicine, Charles University in Prague, Czech Republic
}

Received February 15, 2008

Accepted April 16, 2008

On-line May 13, 2008

\section{Summary}

The processing of species-specific communication signals in the auditory system represents an important aspect of animal behavior and is crucial for its social interactions, reproduction, and survival. In this article the neuronal mechanisms underlying the processing of communication signals in the higher centers of the auditory system - inferior colliculus (IC), medial geniculate body (MGB) and auditory cortex (AC) - are reviewed, with particular attention to the guinea pig. The selectivity of neuronal responses for individual calls in these auditory centers in the guinea pig is usually low - most neurons respond to calls as well as to artificial sounds; the coding of complex sounds in the central auditory nuclei is apparently based on the representation of temporal and spectral features of acoustical stimuli in neural networks. Neuronal response patterns in the IC reliably match the sound envelope for calls characterized by one or more short impulses, but do not exactly fit the envelope for long calls. Also, the main spectral peaks are represented by neuronal firing rates in the IC. In comparison to the IC, response patterns in the MGB and $A C$ demonstrate a less precise representation of the sound envelope, especially in the case of longer calls. The spectral representation is worse in the case of low-frequency calls, but not in the case of broad-band calls. The emotional content of the call may influence neuronal responses in the auditory pathway, which can be demonstrated by stimulation with time-reversed calls or by measurements performed under different levels of anesthesia. The investigation of the principles of the neural coding of species-specific vocalizations offers some keys for understanding the neural mechanisms underlying human speech perception.

\section{Key words}

Vocalization • Guinea pig • Inferior colliculus • Medial geniculate body $\bullet$ Auditory cortex

\section{Corresponding author}

D. Šuta, Department of Auditory Neuroscience, Institute of Experimental Medicine, AS CR, Vídeňská 1083, Prague, Czech Republic. Fax +420 241062787. E-mail: suta@biomed.cas.cz

\section{Introduction}

The processing of communication signals and the spatial localization of a sound source are two key tasks of auditory system function. Communication sounds are a subset of acoustic signals expressed by animals and used in intraspecies interactions. The vocal repertoires of many animal species also include sounds that are not communicative (i.e., are not used in social interactions) but are essential for the behavior of a species, such as sonar signals emitted by echolocating bats that are used to determine the target properties of prey. The central auditory system consists of many nuclei in a hierarchical organization where sensory information is sequentially processed. Individual nuclei of the auditory pathway contribute in a specific way to the processing of complex acoustical signals. This organization reflects the function of the auditory system to extract behaviorally relevant information from a complex acoustic environment using strategies different from those used by other sensory systems.

Understanding the principles of the neural representation of species-specific vocalizations in the 
auditory system may have direct implications for the neural mechanisms underlying human speech perception. Several animal models have recently been used to elucidate how vocalizations are processed in the brain, specifically in the auditory pathway. The most frequently used animals for this type of experiments are songbirds (Theunissen and Shaevitz 2006) and mammals such as bats (Leroy and Wenstrup 2000), rodents (Geissler and Ehret 2004, Wallace et al. 2005), cats (Gourévitch and Eggermont 2007) and primates (Wang 2000). Guinea pigs represent a suitable model for studying the representation of communication signals in the neural system due to their rich repertoire of communication calls and the characteristics of their hearing, with an audible range from $50 \mathrm{~Hz}$ to $50 \mathrm{kHz}$. The functional organization of the guinea pig auditory system and the principles of signal processing in individual auditory nuclei have been extensively studied in our department previously. Our studies were at first focused on single unit responses to simple sound stimuli such as tone and noise bursts (Astl et al. 1996, Syka et al. 2000); later, we became interested in recording multiple unit responses to complex sound stimuli (Šuta et al. 2003, 2007, Syka et al. 2005) and recording unit responses with multichannel electrode probes (Kvašňák et al. 2000b).

The temporal and spike rate-based codes for the processing of the basic spectrotemporal features of communication signals are present at first at the level of the auditory periphery, where auditory nerve fibers faithfully represent fine structures of complex sounds in their temporal discharge patterns (Young and Oertel 2003, for review). At subsequent processing structures along the ascending auditory pathway (i.e., the cochlear nucleus, inferior colliculus, medial geniculate body, and auditory cortex), the upper limit of the temporal representation of repetitive signals gradually decreases due to the biophysical properties of neurons and the temporal integration of converging inputs from one nucleus to the next. A traditional concept in the perception of vocalization signals is based on the existence of highly specific neurons, so-called call detectors. The first studies performed in the auditory cortex of awake squirrel monkeys suggested that neurons might exist in the cortex that extract specific features of calls, similarly to the feature extraction performed in the visual cortex (Wollberg and Newman 1972, Newman and Wollberg 1973, Winter and Funkenstein 1973, Newman and Symmes 1974, Manley and Müller-Preuss 1978, Newman 1978). Later, it became apparent that these neurons could respond to numerous call types, to timereversed calls that had lost their emotional content, or even to artificial complex spectral sounds. It has been suggested that the higher-order processing of vocalizations could be realized by the discharge patterns of neuronal assemblies. In this view, the representation of vocalizations is carried out by dispersed and synchronized cortical cell assemblies that correspond to each individual vocalization in a specific and abstracted way (Rauschecker et al. 1995, Wang et al. 1995). However, several recent studies have again re-opened the hypothesis of a specialization of neurons for very complex sounds. For example, Rauschecker and Tian (2000) found some neurons in the lateral belt of primates responding to whole calls with a full frequency spectrum, but these neurons did not respond to low-pass-filtered or high-pass-filtered calls.

In this paper, data concerning the representation of communication calls in the inferior colliculus (IC), medial geniculate body (MGB) and auditory cortex (AC) of the guinea pig are reviewed with the aim of describing the coding strategies for species-specific vocalizations and their transformation within the auditory system. The results of experiments performed in guinea pigs are discussed against the background of data obtained in other animal species.

\section{Acoustical characteristics of vocalization signals}

With respect to their acoustical pattern, communication sounds are typically complex sounds characterized by time-varying amplitudes and spectral properties. Vocalizations are therefore usually composed of multiple acoustical attributes, and individual neurons within particular nuclei of the auditory pathway may be responsive to a particular vocalization feature(s) or combinations of features. The acoustical patterns of species-specific vocalizations are quite stereotypic, but vocalizations are by their nature stochastic signals and have intrinsic statistical variations for each call type and caller. Statistical analysis of natural sounds and speech reveals that the statistics of spectrotemporal modulations differ from most artificial stimuli used to probe auditory function, including pure tones and white noise (see e.g. Table 1 in Escabí et al. 2003). Many, but not all, of the social communication calls of numerous species are tonal in nature - including those of primates, cats, several species of rodents, bats, birds, and frogs (for review see 
A

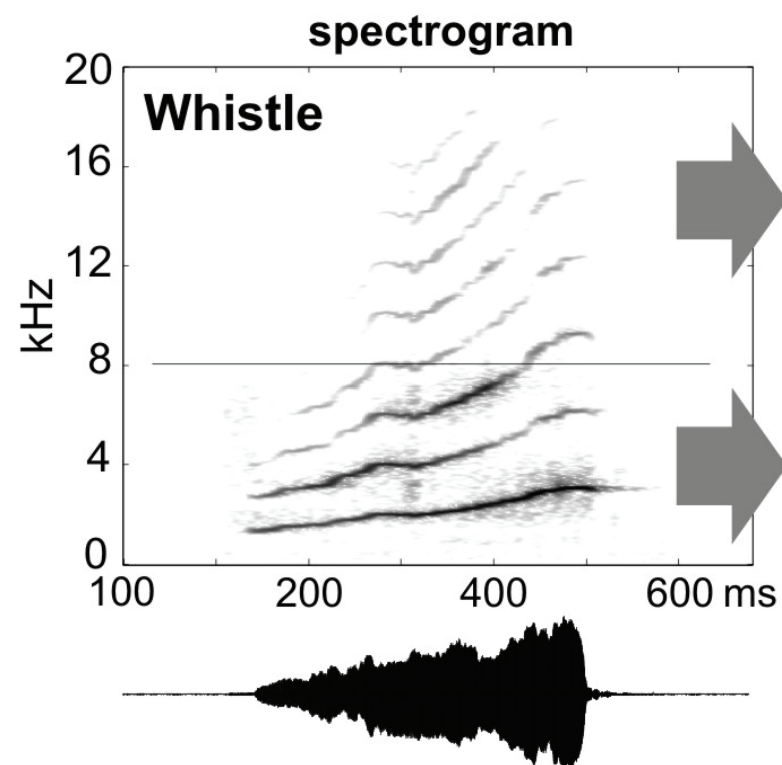

B
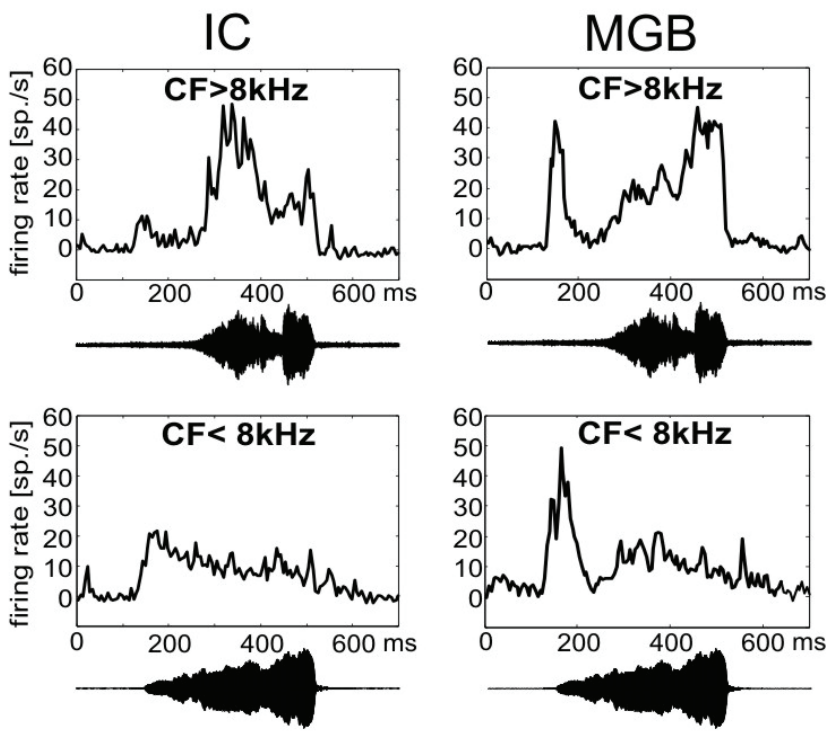

Fig. 1. A. Spectrogram (top) and waveform (bottom) of the guinea pig call whistle. The horizontal line in the spectrogram separates the low-frequency band (below $8 \mathrm{kHz}$ ) and the high-frequency band (above $8 \mathrm{kHz}$ ). B. Average responses (PSTHs) in the IC (left) and MGB (right) are shown separately for neurons with a characteristic frequency below $8 \mathrm{kHz}$ (bottom) and above $8 \mathrm{kHz}$ (top). Each PSTH is accompanied by the waveform of the appropriate frequency component of whistle. Data are adapted from Šuta et al. (2003) for the IC and Suta et al. (2007) for the MGB.

DiMattina and Wang 2006).

In the case of guinea pigs, there are 11 distinct vocalizations within the communication call repertoire (Berryman 1976). Individual calls differ fundamentally in their acoustical features, and the diversity in the acoustical patterns of individual calls is apparent in both the temporal and spectral (frequency) domains. The temporal and spectral parameters of the four most common calls and their variability were described in Syka et al. (1997).

Purr (see Fig. 1A in Šuta et al. 2003) consists of a series of regular, very short impulses $(\sim 30 \mathrm{~ms})$, whereas whistle (Fig. 1A) is a relatively long lasting call ( $\sim 500$ ms) with expressed frequency and intensity modulation. Another difference is in the repetition of individual components; some calls consist of one component (whistle - see Fig. 1A, chirp - see Fig. 2C), but some are characterized by a stereotypic repetition of many components (chutter 1-10 - see Fig. 2B, purr 10-100). The spectral features also vary over a wide range: whistle and chirp are of broad frequency range $(20 \mathrm{kHz})$, but purr and chutter are low-frequency calls below $5 \mathrm{kHz}$.

\section{Inferior colliculus}

The inferior colliculus (IC) is a midbrain auditory structure that integrates information from many ascending auditory pathways, descending corticotectal projections and intercollicular pathways. The processing of information is different in each of the three main subdivisions of the IC - the central nucleus (CNIC), the dorsal cortex (DCIC) and the external cortex (ECIC) which may be distinguished morphologically as well as by different inputs and outputs. Syka et al. (2000) reported the response properties of neurons in the guinea pig in response to tonal and noise stimuli: in comparison with DCIC and ECIC neurons, CNIC neurons as a group were characterized by a sharper frequency tuning, a lower average threshold, a shorter average first-spike latency of response to tones at the characteristic frequency $(\mathrm{CF})$, a higher occurrence of non-monotonic rate/level functions and a higher rate of spontaneous activity. Aitkin et al. (1994) pointed to differences between subdivisions of the IC in the cat apparent in responses to vocal stimuli, which were more effective in terms of higher firing rates than noise or $\mathrm{CF}$ stimuli in $27 \%$ of units in the CNIC, but in $82 \%$ in the ECIC and $72 \%$ in the DCIC. There were no units that responded exclusively to one vocal stimulus, but a high proportion of units in the ECIC responded strongly to broad-band stimuli, and some of these showed clear preferences for one vocal stimulus over others.

Neuronal responses in the CNIC of the guinea pig to simple stimuli (tones, noise bursts) demonstrate predominantly a sustained response pattern, but a 
significant portion of phasic neurons ( $\sim 30 \%$ according to Syka et al. 2000) is present in the IC as well. They respond typically by an onset firing pattern at the beginning of the stimulus. The selectivity of guinea pig IC units for individual calls is very low, as the majority of units respond to all four used calls and the coding is rather based on coding of the spectrotemporal pattern of the speciesspecific vocalization by discharge patterns of the IC neuronal responses (Šuta et al. 2003). The temporal envelope of the call is coded by the firing rate, except for the slow modulation of whistle (Fig. 1). Individual peristimulus time histograms (PSTHs), and especially average PSTHs, reliably copy the envelope of calls characterized by one or more short phrases (i.e., chirp, chutter, and purr; see Fig. 2 for the later two calls). In calls containing more than one component (phrase), such as purr and chutter, the acoustical patterns of these components are very stereotypic. Also, the individual peaks in the response pattern are very stereotypic with the exception of the first (onset) peak in the response to purr, which is enhanced more than would be expected from the acoustical pattern. Some variability in the peak amplitude of the sound is reflected in the response, given that a stronger response correlates with a greater intensity of the phrase.

The response seems to omit slow modulation of the sound envelope. This phenomenon is present mainly in the response to whistle, in which the sustained character of the response indicates only the presence of energy, but the slow changes in the sound envelope are not reflected in the modulations of the firing rate. This inability of units to follow the slow fluctuations in the envelope corresponds well with the weak synchronization between neuronal discharge and sound envelope as seen for sinusoidal amplitude-modulated tones at low modulation frequencies. The modulation transfer function of IC units typically has a band-pass character as shown by Rees and Moller (1983) in the rat and later by Rees and Palmer (1989) in the guinea pig.

The importance of the temporal structure of the call is demonstrated by the fact that whistle evoked a stronger response (by $24 \%$ ) in the guinea pig than did the artificial, time-reversed whistle (Šuta et al. 2003). However, not every unit responded in this manner; there were neurons with a significantly stronger response to the natural ("forward-selective," $30 \%$ ) whistle as well as to the time-reversed whistle ("reverse-selective," $15 \%$ ).

Rate coding of the sound frequency spectrum demonstrates, in principle, whether the high intensity of a particular sound frequency band is reflected in an elevated firing rate of neurons having their CFs near that particular frequency and, vice versa, whether a lack of energy in a frequency band results in no response of neurons with the appropriate CF. This type of analysis in the form of rate versus CF plots (Fig. 3), where the average response of units with a particular $\mathrm{CF}$ is calculated as an equivalent of the intensity of the appropriate spectral component in the stimulus, identifies some of the major spectral components of the sound. The spectral profile calculated from the response does not exactly fit the short-term spectrum, but it clearly marks the position of the main spectral peaks. There are two main discrepancies: in the relative magnitudes of the spectral peaks and in the representation of the higher harmonics. In some cases, at first the magnitudes of the sound spectral peaks are not exactly matched by the magnitudes of the peaks in the response profile. The magnitudes of the peaks in the low-CF region are lowered by inhibition, which predominantly occurs in low-CF units, and also the pattern of temporal modulation may affect the spectral profile because an onset firing of units within a particular CF range can be greater than a sustained firing within another $\mathrm{CF}$ range. Later, higher harmonics are not represented by several individual peaks but rather by a complex peak.

Several studies have demonstrated that not only the frequency spectrum, but also the pattern of its modulation are important aspects in the processing of vocalizations. Andoni et al. (2007) showed that most species-specific calls of Mexican free-tailed bats have downward-sweeping frequency-modulated (FM) components with sweep velocities that correspond with the preferred sweep velocities of IC neurons and hypothesized that this close quantitative correspondence among the features of signals and responses may suggest that IC cells are tuned by inhibition to respond optimally to spectral motion cues present in their conspecific vocalizations. Similarly, Kao et al. (1997) demonstrated in the rat IC that for some FM-sensitive neurons, the FM response may account for their responses to vocalization sounds.

In principle, the IC provides the encoding of the spectrotemporal acoustic patterns of vocalizations by IC units in such a way that the presence or absence of neural responses is a consequence of the tuning properties of the IC units and of the spectrotemporal acoustical pattern of the sound. Neuronal response patterns represent the temporal pattern of calls (except for slow modulation) and the dominant spectral components of calls. 
A

\section{$\%$ Response types}
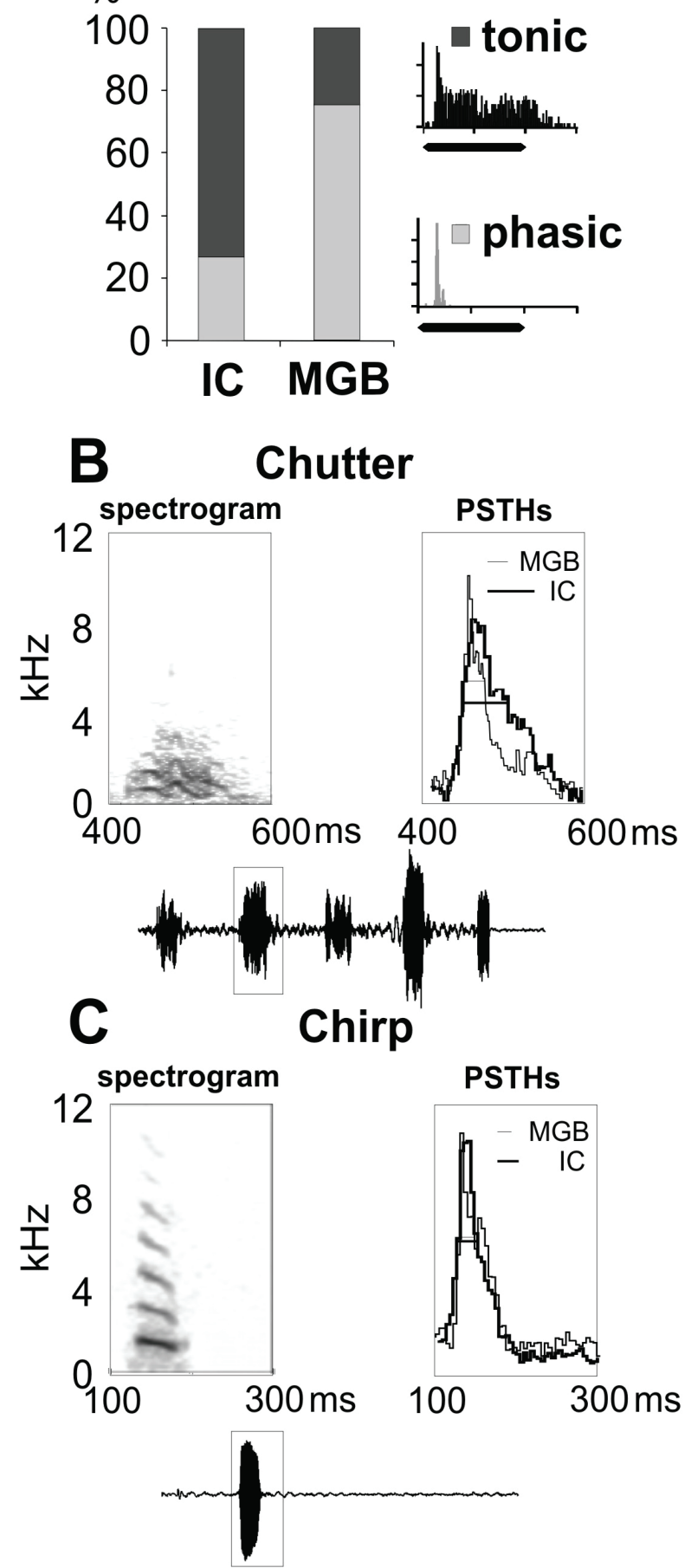

\section{Medial geniculate body}

The MGB represents the thalamic part of the auditory pathway, receiving projections from the IC and providing projections to the cerebral cortex. Its structure is non-homogeneous with three subdivisions, of which the ventral division is considered to be a specific auditory relay projecting to the AI area of the auditory cortex. In
Fig. 2. A. The percentage of neurons characterized as tonic and phasic according to their response to pure tones is compared for the IC and MGB. Data for the IC are adapted from Syka et al. (2000) and data for the MGB from Kvašnák et al. (2000a). B. The spectrogram of one component of chutter (marked by the box in the chutter waveform on bottom panel) is shown in the top left panel whereas the top right panel compares the average response in the IC (bold) and MGB (thin). Horizontal bars illustrate the response duration measured at $50 \%$ of the maximal response in the IC and MGB. C. Bottom panel shows the waveform of chirp. The spectrogram of chirp (marked by the box in the chirp waveform) is shown in the top left panel whereas the top right panel compares the average response in the IC (bold) and MGB (thin). Data for plots B. and C. are adapted from Šuta et al. (2003) for the IC and Šuta et al. (2007) for the MGB.

contrast to the IC, neuronal responses to simple stimuli such as tones or broad-band noise are predominantly of a phasic type (Fig. 2) with expressed response to the onset of stimulus (Kvašňák et al. 2000a, Wallace et al. 2007).

The transformation of neuronal responses in the MGB is apparent also in responses to communication calls. Šuta et al. (2007) demonstrated various levels of fidelity in temporal envelope representation - highly accurate in the case of calls with a rapidly modulated temporal structure such as chirp (Fig. 2C), but less precise for slow amplitude modulation as in the case of chutter (Fig. 2B) or whistle. Cells in both the ventral and medial divisions of the MGB showed a response that phase-locked to the fundamental frequency of the guinea pig purr and may be involved in analyzing communication calls (Wallace et al. 2007).

The neuronal response patterns in the MGB demonstrated a strong dependence of the firing pattern of the units on the spectral composition of the call (Fig. 1). A comparison between the sound spectrum features and the rate vs. CF profile (where neuronal firing is assigned according to the neuronal $\mathrm{CF}$ ) typically indicated the dominant spectral components, but in some cases some local spectral peaks were intensified and created dominant elements in the rate vs. CF profiles (Šuta et al. 2007). This corresponds to the observation of Tanaka and Taniguchi's (1991) that the responses to vocalizations in the MGB may display discharge patterns that are not possible to predict from the properties of their responses to pure tones. Quantitative assessment demonstrated that in the case of call spectrum representation, the representation of the spectral features is preserved in the MGB for wideband calls similarly as in the IC, but it is less precise in the case of low-frequency calls.

A study of the functional connections between neurons within the MGB using cross-correlation analysis by Kvašñák et al. (2000b) showed that in some neurons 

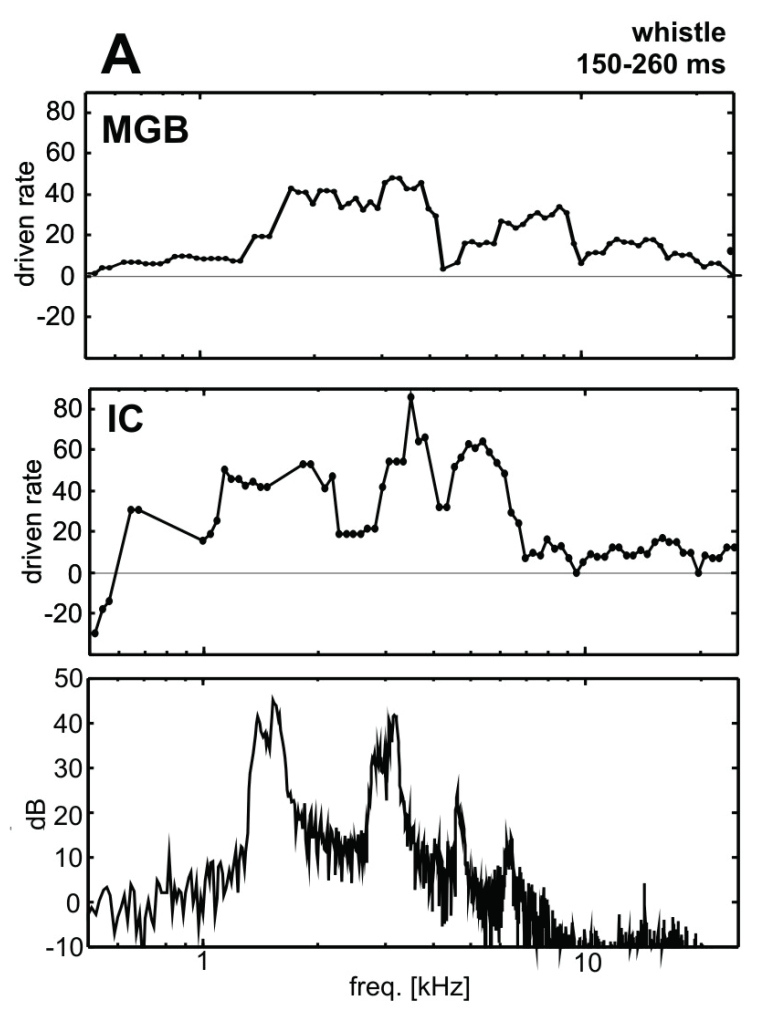
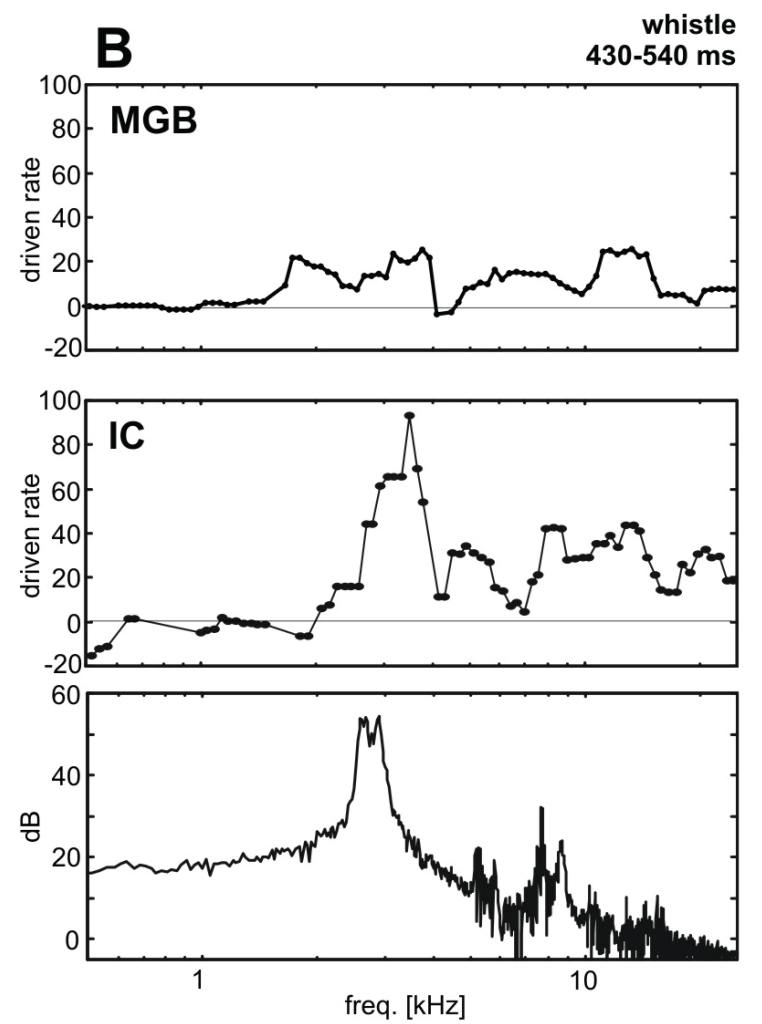

Fig. 3. Comparison of sound short-time frequency spectra (bottom) with a neuronal rate vs. CF profile in the IC (middle) and in the MGB (top) for two different time-intervals of whistle. Each point in the rate vs. CF profile represents the average response ( $y$-axis) of all neurons with a CF within \pm 0.175 octave from the particular CF (x-axis). A. A lack of sound energy below $1 \mathrm{kHz}$ and above $10 \mathrm{kHz}$ is reflected in a weak or absent response of neurons with a CF within this range in both the MGB and IC. B. No response of neurons with a CF $<2 \mathrm{kHz}$ corresponds with the lack of energy in the call in this frequency range. Data adapted from Šuta et al. (2005, 2007).

(15\% of pairs) the change from one acoustical stimulus to another resulted in a change in the functional connection, which suggests that not only rate coding mechanisms as discussed above, but also neuronal synchrony may be employed in stimulus encoding at the level of the MGB.

Our results (Šuta et al. 2007) indicated that the firing rate for a natural whistle is not dramatically enhanced in comparison to a time-reversed one in the MGB of the guinea pig. The difference of $13 \%$ on average is lower than that found in the IC under comparable conditions (Šuta et al. 2003). Even weaker preference was reported in the study by Philibert et al. (2005), who compared the responses of auditory thalamus neurons in the guinea pig and rat to guinea pig vocalizations. The authors concluded that the thalamic neurons of guinea pigs and rats displayed a similar response strength to guinea pig vocalizations and did not exhibit a significant preference for the natural over the time-reversed version of the calls in either species.

The prevailing onset character of the neuronal responses to acoustical stimuli in the MGB significantly influences the character of MGB neuronal responses to vocalization signals. The representation of individual calls is affected to a different extent according to the different spectral and temporal features of individual calls - a less precise representation is present in the case of the temporal envelope for longer calls and of the spectral envelope for narrow-band calls.

\section{Auditory cortex}

The core area of the auditory cortex $(\mathrm{AC})$ in the guinea pig consists of the primary $\mathrm{AC}(\mathrm{AI})$ and the dorsal field (DC), which are surrounded by several belt areas (Wallace et al. 2000). A detailed study of the cortical responses to the guinea pig repertoire of calls is, at the present time, lacking, but several studies (mostly based on reponses to selected call samples) suggest a similarity between the responsiveness of the AI and the vMGB to some extent. The original description of the relationship between the MGB and $\mathrm{AC}$ in the processing of vocalizations comes from Creutzfeldt et al. (1980). They analyzed synaptically connected neurons in the MGB and 
AI in unanesthetized guinea pigs and reported that all neurons in both the MGB and AI responded to a variety of natural calls of the same or of other species. The authors found that cortical neurons responded to pure tones more frequently with an onset response than did the corresponding neurons in the MGB. However, the responses of MGB cells to a call stimulation consisted of more components than the responses of cortical cells. Cortical neurons responded less precisely to calls consisting of a train of pulses; the repetitive elements of a call were not represented in cortical cell responses if the repetition rate was too high. On the other hand, Wallace et al. (2005) reported that the temporal structure of a train of pulses of the guinea pig call "purr" was represented with the same fidelity by the low-frequency cortical cells as by the low-frequency cells of the MGB.

The parameters of responses are not homogenously distributed within the auditory cortex. Neurons in the dorsal belts of the guinea pig AC respond more strongly to broad-band stimuli than to pure tones, whereas neurons in the ventral belts respond better to pure tones, have longer onset latencies and give a more sustained response than do units in the AI (Wallace et al. 2000). The non-homogenous distribution of the properties of neuronal receptive fields has been shown within the primary AC in the cat (Schreiner 1998). Neuronal receptive fields were interpreted as forming maps that represent specific stimulus information in a topographic way. The distribution of activity in the AI for elemental speech sounds had a patchy temporal-spatial pattern with multiple foci of activity. Non-homogenous responses within the guinea pig AC have been recently suggested by Šuta et al. (2008), who showed that neuronal responses to calls consisting of several phrases (purr, chutter) were increasingly synchronized in the ventro-dorsal direction in the AI. Responses to vocalizations (chutter and whistle) may also vary between cortical layers. Wallace and Palmer (2008) demonstrated in guinea pigs that the response to the first phrase of chutter was smaller and occurred about $10 \mathrm{~ms}$ later in the deep layers in comparison with layers II and III, whereas the response to whistle was strongest in the deep layers of AI.

Neuronal responses in the AC in particular cases resemble those in the $\mathrm{MGB}$, but recent studies have shown that, in principle, the auditory cortex forms representations of complex sounds that are no longer faithful replicas of their acoustic structures (Wang 2007).

\section{Effects of anesthesia}

The majority of data presented above were obtained in anesthetized animals. Anesthesia is an important factor that can significantly influence sensory processing in the brain centers, therefore we must be aware of its effects and must consider its possible influence on the relevancy of the experimental data. Many previous papers have reported mainly a suppressive effect of various anesthetics on spontaneous activity in different cortical and subcortical nuclei (Evans and Nelson 1973, Bock and Webster 1974, Kuwada et al. 1989, Zurita et al. 1994), but much less is known about the impact of anesthetics on sound-evoked activity in the auditory system and on signal processing in the neuronal circuits. Several authors have demonstrated that the depth of anesthesia can modulate the variability of stimulusevoked responses, the strength of inhibition, the type of response or the tuning properties of neurons (Kisley and Gerstein 1999, Gaese and Ostwald 2001, Anderson and Young 2004). However, individual anesthetics can differ in their effect on neuronal activity (Cohen and Britt 1982, Astl et al. 1996). IC neurons in animals anesthetized with a ketamine-xylazine mixture had a higher spontaneous firing rate, more frequently expressed sustained responses and had lower response thresholds than neurons recorded in animals under pentobarbital or urethane anesthesia (Astl et al. 1996).

The effects of anesthesia seem to be important when studying the processing of complex sounds, mainly species-specific vocalizations that have a high behavioral impact. Whereas the effects of anesthesia on neuronal responses to simple acoustical stimuli are mostly suppressive, the effects of anesthesia on responses evoked by species-specific vocalizations are much more variable. The administration of anesthesia may increase or modify the response to some types of calls and suppress the response to others. A wide variety of response patterns to acoustical stimuli, in comparison with awake anaesthetized animals, was found in experiments in which single units were recorded in the auditory cortex of non-anaesthetized cats (Gerstein and Kiang 1964, Evans and Whitfield 1964) or rats (Gaese and Ostwald 2003). Elhilali et al. (2002) found more complex receptive field shapes, more complex spectral processing and increased selectivity in the direction of frequency modulation in cortical neurons recorded in awake ferrets than in ketamine-anesthetized animals. The results of our previous experiments (Syka et al. 2005) 
demonstrated a significant influence of ketamine-xylazine anesthesia on the responses of neurons in the auditory cortex of the guinea pig to species-specific vocalizations. The effect was, however, not uniform since the administration of anesthesia was able to increase the response of a neuron to some types of stimuli and suppress the response of the same neuron to others. It should be emphasized that a suppressive effect of anesthesia was more frequently seen than was response augmentation. Not only the response strength, but also the temporal pattern of the response to vocalization calls was changed in some cases under anesthesia, which may indicate a diversity in the impact of anesthesia on the synaptic inputs of the recorded neurons. The effect of anesthesia on the processing of acoustical information was evident not only at the cortical level, but also at the subcortical stages of the auditory system such as the cochlear nucleus (Anderson and Young 2004), inferior colliculus (Kuwada et al. 1989, Astl et al. 1996, Torterolo et al. 2002) or medial geniculate body (Cotillon-Williams and Edeline 2003, Massaux et al. 2004). Therefore, the modification of cortical activity can result not only from the direct effect of an anesthetic agent on intracortical processing, but also from modified subcortical activity or, most likely, from a combination of subcortical and intracortical effects.

Anesthesia has a significant effect on neuronal activity and influences the rate as well as the pattern of neuronal response; this argues for the use of awake and unrestrained animals, which, while respecting all ethical aspects of experimental work, seems to be an important step in adequately addressing key issues such as the neural representation of complex acoustical signals.

\section{Conclusions}

Recent findings indicate that many principles of the processing of complex acoustical stimuli in humans are shared among many species of mammals, which can be illustrated by a general decrease in the highest amplitude modulation frequencies that influence the neural response, either in terms of average rate or synchronization, as one records at higher and higher levels along the neuraxis (Joris et al. 2004) or by hemispheral asymmetries in the processing of complex stimuli (Wetzel et al. 1998, Rybalko et al. 2006, Zatorre and Belin 2001). Although the data cited in this article are largely based on studies in the guinea pig, the neural coding strategies discussed here are likely applicable to the auditory system of other mammals, and data from the guinea pig model can be compared to studies performed in other species that describe the transformation of the neural representations of vocalizations and other complex stimuli from the IC to the AC (for example, by Bartlett and Wang 2007, in primates).

\section{Conflict of Interest}

There is no conflict of interest.

\section{Acknowledgements}

The study was supported by grants GACR 309/07/1336, AVOZ50390512 and LC 554.

\section{References}

AITKIN L, TRAN L, SYKA J: The responses of neurons in subdivisions of the inferior colliculus of cats to tonal, noise and vocal stimuli. Exp Brain Res 98: 53-64, 1994.

ANDERSON MJ, YOUNG ED: Isoflurane/ $\mathrm{N}_{2} \mathrm{O}$ anesthesia suppresses narrowband but not wideband inhibition in dorsal cochlear nucleus. Hear Res 188: 29-41, 2004.

ANDONI S, LI N, POLLAK GD: Spectrotemporal receptive fields in the inferior colliculus revealing selectivity for spectral motion in conspecific vocalizations. J Neurosci 27: 4882-4893, 2007.

ASTL J, POPELÁŘ J, KVAŠŇÁK E, SYKA J: Comparison of response properties of neurons in the inferior colliculus of guinea pigs under different anesthetics. Audiology 35: 335-345, 1996.

BARTLETT EL, WANG X: Neural representations of temporally modulated signals in the auditory thalamus of awake primates. J Neurophysiol 97: 1005-1017, 2007.

BERRYMAN JC. Guinea-pig vocalizations: their structure, causation and function. Z Tierpsychol 41: 80-106, 1976.

BOCK GR, WEBSTER WR: Spontaneous activity of single units in the inferior colliculus of anesthetized and unanesthetized cats. Brain Res 76: 150-154, 1974.

COHEN MS, BRITT RH: Effects of sodium pentobarbital, ketamine, halothane, and chloralose on brainstem auditory evoked responses. Anesth Analg 61: 338-343, 1982. 
COTILLON-WILLIAMS N, EDELINE JM: Evoked oscillations in the thalamo-cortical auditory system are present in anesthetized but not in unanesthetized rats. J Neurophysiol 89: 1968-1984, 2003.

CREUTZFELDT OD, HELLWEG F-C, SCHREINER CE: Thalamocortical transformation of responses to complex auditory stimuli. Exp Brain Res 39: 87-104, 1980.

DiMATTINA C AND WANG. X: Virtual vocalization stimuli for investigating neural representations of speciesspecific vocalizations. J Neurophysiol 95: 1244-1262, 2006.

ELHILALI M, FRITZ JB, BOZAK D, DEPIREUX DA, SHAMMA SA: Comparison of response characteristics in auditory cortex of the awake and anesthetized ferret. Assoc Res Otolaryngol Abst. 162, 2002.

ESCABÍ MA, MILLER LM, READ HL, SCHREINER CE: Naturalistic auditory contrast improves spectrotemporal coding in the cat inferior colliculus. $J$ Neurosci 23: 11489-11504, 2003.

EVANS EF, NELSON PG: The responses of single neurones in the cochlear nucleus of the cat as a function of their location and the anaesthetic state. Exp Brain Res 17: 402-427, 1973.

EVANS EF, WHITFIELD IC: Classification of unit responses in the auditory cortex of the unanaesthetized and unrestrained cat. $J$ Physiol Lond 171: 476-493, 1964.

GAESE BH, OSTWALD J: Anesthesia changes frequency tuning of neurons in the rat primary auditory cortex. J Neurophysiol 86: 1062-1066, 2001.

GAESE BH, OSTWALD J: Complexity and temporal dynamics of frequency coding in the awake rat auditory cortex. Eur J Neurosci 18: 2638-2652, 2003.

GEISSLER DB, EHRET G: Auditory perception vs. recognition: representation of complex communication sounds in the mouse auditory cortical fields. Eur J Neurosci 19: 1027-1040, 2004.

GERSTEIN GL, KIANG NY: Responses of single units in the auditory cortex. Exp Neurol 10: 1-18, 1964.

GOURÉVITCH B, EGGERMONT JJ: Spatial representation of neural responses to natural and altered conspecific vocalizations in cat auditory cortex. J Neurophysiol 97: 144-158, 2007.

JORIS PX, SCHREINER CE, REES A: Neural processing of amplitude-modulated sounds. Physiol Rev 84: 541-577, 2004.

KAO MC, POON PW, SUN X: Modeling of the response of midbrain auditory neurons in the rat to their vocalization sounds based on FM sensitivities. Biosystems 40: 103-109, 1997.

KISLEY MA, GERSTEIN GL: Trial-to-trial variability and state-dependent modulation of auditory evoked responses in cortex. J Neurosci 19: 10451-10460, 1999.

KUWADA S, BATRA R, STANFORD TR: Monaural and binaural response properties of neurons in the inferior colliculus of the rabbit: effects of sodium pentobarbital. J Neurophysiol 61: 269-282, 1989.

KVAŠŇÁK E, POPELÁŘ J, SYKA J: Discharge properties of neurons in subdivisions of the medial geniculate body of the guinea pig. Physiol Res 49: 369-378, 2000a.

KVAŠŇÁK E, ŠUTA D, POPELÁŘ J, SYKA J: Neuronal connections in the medial geniculate body of the guinea-pig. Exp Brain Res 132: 87-102, 2000b.

LEROY SA, WENSTRUP JJ: Spectral integration in the inferior colliculus of the mustached bat. $J$ Neurosci 20: 8533$8541,2000$.

MANLEY JA, MÜLLER-PREUSS P: Response variability of auditory cortex cells in the squirrel monkey to constant acoustic stimuli. Exp Brain Res 32:171-180, 1978.

MASSAUX A, DUTRIEUX G, COTILLON-WILLIAMS N, MANUNTA Y, EDELINE JM: Auditory thalamus bursts in anesthetized and non-anesthetized states: contribution to functional properties. J Neurophysiol 91: 21172134, 2004.

NEWMAN JD: Perception of sounds used in species-specific communication: the auditory cortex and beyond. $J$ Med Primatol 7: 98-105, 1978.

NEWMAN JD, SYMMES D: Arousal effects on unit responsiveness to vocalizations in squirrel monkey auditory cortex. Brain Res 78: 125-138, 1974.

NEWMAN JD, WOLLBERG Z: Multiple coding of species-specific vocalizations in the auditory cortex of squirrel monkeys. Brain Res 54: 287-304, 1973.

PHILIBERT B, LAUDANSKI J, EDELINE JM: Auditory thalamus responses to guinea-pig vocalizations: a comparison between rat and guinea-pig. Hear Res 209: 97-103, 2005. 
RAUSCHECKER JP, TIAN B: Mechanisms and streams for processing of "what" and "where" in auditory cortex. Proc Natl Acad Sci USA 97: 11800-11806, 2000.

RAUSCHECKER JP, TIAN B, HAUSER M: Processing of complex sounds in the macaque nonprimary auditory cortex. Science 268: 111-114, 1995.

REES A, MØLLER AR: Responses of neurons in the inferior colliculus of the rat to AM and FM tones. Hear Res 10: 301-330, 1983.

REES A, PALMER AR: Neuronal responses to amplitude-modulated and pure-tone stimuli in the guinea pig inferior colliculus, and their modification by broadband noise. J Acoust Soc Am 85: 1978-1994, 1989.

RYBALKO N, ŠUTA D, NWABUEZE-OGBO F, SYKA J: Effect of auditory cortex lesions on the discrimination of frequency-modulated tones in rats. Eur J Neurosci 23: 1614-1622, 2006.

SCHREINER CE: Spatial distribution of responses to simple and complex sounds in the primary auditory cortex. Audiol Neurootol 3: 104-122, 1998.

ŠUTA D, KVAŠŇÁK E, POPELÁŘ J, SYKA J: Representation of species-specific vocalizations in the inferior colliculus of the guinea pig. J Neurophysiol 90: 3794-3808, 2003.

ŠUTA D, POPELÁŘ J, KVAŠŇÁK E, SYKA J: Representation of species-specific vocalizations in the medial geniculate body of the guinea pig. Exp Brain Res 183: 377-388, 2007.

ŠUTA D, POPELÁ Ǩ J, BURIANOVÁ J, SYKA J: Neuronal responses to vocalization signals in the auditory cortex of the guinea pig. Assoc Res Otolaryngol Abstr 906, 2008.

SYKA J, POPELÁŘ J, KVAŠŇÁK E, ŠUTA D, JILEK M: Processing of species-specific vocalizations in the inferior colliculus and medial geniculate body of the guinea pig. In Acoustical Signal Processing in the Central Auditory System. J SYKA (ed), Plenum Press, New York, 1997, pp 431-441.

SYKA J, POPELÁŘ J, KVAŠŇÁK E, ASTL J: Response properties of neurons in the central nucleus and external and dorsal cortices of the inferior colliculus in guinea pig. Exp Brain Res 133: 254-266, 2000.

SYKA J, ŠUTA D, POPELÁŘ J: Responses to species-specific vocalizations in the auditory cortex of awake and anesthetized guinea pigs. Hear Res 206: 177-184, 2005.

TANAKA H AND TANIGUCHI I: Responses of medial geniculate neurons to species-specific vocalized sounds in the guinea pig. Jpn J Physiol 41: 817-829, 1991.

THEUNISSEN FE, SHAEVITZ SS: Auditory processing of vocal sounds in birds. Curr Opin Neurobiol 16: 400-407, 2006.

TORTEROLO P, FALCONI A, MORALES-COBAS G, VELLUTI RA: Inferior colliculus unitary activity in wakefulness, sleep and under barbiturates. Brain Res 935: 9-15, 2002.

WALLACE MN, RUTKOWSKI RG, PALMER AR: Identification and localisation of auditory areas in guinea pig cortex. Exp Brain Res 132: 445-456, 2000.

WALLACE MN, ANDERSON LA, PALMER AR: Phase-locked responses to pure tones in the auditory thalamus. J Neurophysiol 98: 1941-1952, 2007.

WALLACE MN, PALMER AR: Laminar differences in the response properties of cells in the primary auditory cortex. Exp Brain Res 184: 179-191, 2008.

WALLACE MN, SHACKLETON TM, ANDERSON LA, PALMER AR: Representation of the purr call in the guinea pig primary auditory cortex. Hear Res 204: 115-126, 2005.

WANG X: On cortical coding of vocal communication sounds in primates. Proc Natl Acad Sci USA 97: 11843-11849, 2000.

WANG X: Neural coding strategies in auditory cortex. Hear Res 229: 81-93, 2007.

WANG X, MERZENICH MM, BEITEL R, SCHREINER CE: Representation of a species-specific vocalization in the primary auditory cortex of the common marmoset: temporal and spectral characteristics. $J$ Neurophysiol $\mathbf{7 4}$ : 2685-2706, 1995.

WETZEL W, OHL FW, WAGNER T, SCHEICH H: Right auditory cortex lesion in Mongolian gerbils impairs discrimination of rising and falling frequency-modulated tones. Neurosci Lett 252: 115-118, 1998.

WINTER P, FUNKENSTEIN HH: The effect of species-specific vocalization on the discharge of auditory cortical cells in the awake squirrel monkey (Saimiri sciureus). Exp Brain Res 18: 489-504, 1973. 
WOLLBERG Z, NEWMAN JD: Auditory cortex of squirrel monkey: response patterns of single cells to speciesspecific vocalizations. Science 175: 212-214, 1972.

YOUNG ED, OERTEL D: The cochlear nucleus. In: Synaptic Organization of the Brain, GM SHEPHERD (ed), Oxford University Press, New York, 2003, pp 125-163.

ZATORRE RJ, BELIN P: Spectral and temporal processing in human auditory cortex. Cerebr Cortex 11: 946-953, 2001.

ZURITA P, VILLA AE, DE RIBAUPIERRE Y, DE RIBAUPIERRE F, ROUILLER EM: Changes of single unit activity in the cat's auditory thalamus and cortex associated to different anesthetic conditions. Neurosci Res 19: 303-316, 1994. 